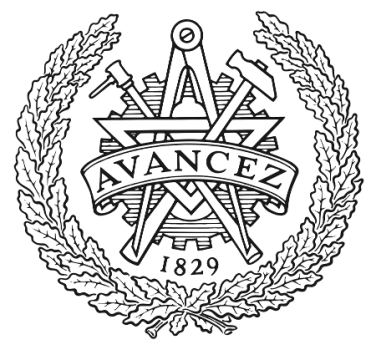

CHALMERS

UNIVERSITY OF TECHNOLOGY

\title{
Phantom motor execution facilitated by machine learning and augmented reality as treatment for phantom limb pain: a single group, clinical trial in
}

Downloaded from: https://research.chalmers.se, 2023-04-26 13:00 UTC

Citation for the original published paper (version of record):

Ortiz Catalan, M., Gudmundsdottir, R., Kristoffersen, M. et al (2016). Phantom motor execution facilitated by machine learning and augmented reality as treatment for phantom limb pain: a single group, clinical trial in patients with chronic intractable phantom limb pain. The Lancet, 388(10062): 2885-2894. http://dx.doi.org/10.1016/s0140-6736(16)31598-7

N.B. When citing this work, cite the original published paper. 


\section{Phantom motor execution facilitated by machine learning and augmented reality as treatment for Phantom Limb Pain}

Max Ortiz-Catalan, Ph.D. ${ }^{1,2,3 \S}$, Rannveig A. Guđmundsdóttir, M.Sc. ${ }^{1,3}$, Morten B. Kristoffersen, M.Sc. ${ }^{1,3}$, Alejandra Zepeda-Echavarria, B.Sc. ${ }^{1,3}$, Kerstin Caine-Winterberger, O.T.R. ${ }^{2}$, Katarzyna Kulbacka-Ortiz, M.Sc. ${ }^{2}$, Cathrine Widehammar, M.Sc. ${ }^{4,5}$, Karin Eriksson, B.Sc. ${ }^{5}$, Anita Stockselius, O.T.R. ${ }^{6}$, Christina Ragnö O.T.R. ${ }^{6}$, Zdenka Pihlar, O.T.R. ${ }^{7}$, Prof. Helena Burger, M.D. ${ }^{7}$, and Prof. Liselotte Hermansson, Ph.D. ${ }^{4,8}$.

${ }^{1}$ Department of Signals and Systems, Chalmers University of Technology, Hörsalsvägen 11, SE - 41296 , Gothenburg, Sweden.

${ }^{2}$ Centre for Advanced Reconstruction of Extremities (C.A.R.E.), Sahlgrenska University Hospital, Göteborgsvägen 31, 43180 Mölndal, Sweden.

3 Integrum AB, Krokslätts Fabriker 50, SE-431 37, Mölndal, Sweden.

${ }^{4}$ University Health Care Research Centre, Faculty of Medicine and Health, Örebro University, Box 1613 SE-701 16, Örebro, Sweden.

${ }^{5}$ Department of Pediatrics, Faculty of Medicine and Health, Örebro University, Box 1613, SE-701 16, Örebro, Sweden.

${ }^{6}$ BräckeDiakoni Rehabcenter Sfären, Bockholmsvägen 1, 170 78, Solna, Sweden.

7 University Rehabilitation Institute, Linhartova 51, 1000, Ljubljana, Slovenia.

8 Department of Prosthetics and Orthotics, Faculty of Medicine and Health, Örebro University, Box 1613 SE-701 16, Örebro, Sweden.

The following is the accepted version of the manuscript made available under the CC-BY-NC-ND 4.0 license.

Please see the formal publication: http://dx.doi.org/10.1016/s0140$\underline{6736(16) 31598-7}$

(c) 2016. This manuscript version is made available under the CC-BY-NCND 4.0 license http://creativecommons.org/licenses/by-nc-nd/4.0/ 


\section{Phantom motor execution facilitated by machine learning and augmented reality as treatment for Phantom Limb Pain}

Max Ortiz-Catalan, Ph.D. ${ }^{1,2,3 \S}$, Rannveig A. Guđmundsdóttir, M.Sc. ${ }^{1,3}$, Morten B. Kristoffersen, M.Sc. ${ }^{1,3}$, Alejandra Zepeda-Echavarria, B.Sc. ${ }^{1,3}$, Kerstin Caine-Winterberger, O.T.R. ${ }^{2}$, Katarzyna Kulbacka-Ortiz, M.Sc. ${ }^{2}$, Cathrine Widehammar, M.Sc. ${ }^{4,5}$, Karin Eriksson, B.Sc. ${ }^{5}$, Anita Stockselius, O.T.R. ${ }^{6}$, Christina Ragnö O.T.R. ${ }^{6}$, Zdenka Pihlar, O.T.R. ${ }^{7}$, Prof. Helena Burger, M.D. ${ }^{7}$, and Prof. Liselotte Hermansson, Ph.D. ${ }^{4,8}$.

${ }^{1}$ Department of Signals and Systems, Chalmers University of Technology, Hörsalsvägen 11, SE - 41296 , Gothenburg, Sweden.

${ }^{2}$ Centre for Advanced Reconstruction of Extremities (C.A.R.E.), Sahlgrenska University Hospital, Göteborgsvägen 31, 43180 Mölndal, Sweden.

${ }^{3}$ Integrum AB, Krokslätts Fabriker 50, SE-431 37, Mölndal, Sweden.

${ }^{4}$ University Health Care Research Centre, Faculty of Medicine and Health, Örebro University, Box 1613 SE-701 16, Örebro, Sweden.

${ }^{5}$ Department of Pediatrics, Faculty of Medicine and Health, Örebro University, Box 1613, SE-701 16, Örebro, Sweden.

${ }^{6}$ BräckeDiakoni Rehabcenter Sfären, Bockholmsvägen 1, 170 78, Solna, Sweden.

7 University Rehabilitation Institute, Linhartova 51, 1000, Ljubljana, Slovenia.

8 Department of Prosthetics and Orthotics, Faculty of Medicine and Health, Örebro University, Box 1613 SE-701 16, Örebro, Sweden.

$\S$ Corresponding author: Dr. Max Ortiz Catalan, Assist. Professor, Chalmers University of Technology, Department of Signals and Systems, Hörsalsvägen 11, SE-41296, Gothenburg, Sweden. Phone: +46317725149. E-mail: maxo@chalmers.se

\section{Abstract}

Background: Phantom limb pain (PLP) is a debilitating condition for which no effective treatment has been found. We hypothesized that reengagement of central and peripheral circuitry involved in motor execution could reduce PLP via competitive plasticity and reversal of cortical reorganization.

Methods: Fourteen patients with intractable chronic PLP, for whom conventional treatments failed, received 12 sessions of phantom motor execution in four hospitals using machine learning and augmented/virtual reality (AR/VR). Changes in intensity, frequency, duration, quality, and intrusion of PLP were assessed using the numeric rating scale (NRS), the pain rating index (PRI), and the weighted pain distribution (WPD) scale. Changes in medication and prostheses were also monitored. Follow-up interviews were done at 1,3 , and 6 months after the last session.

Results: After twelve sessions patients showed statistically and clinically significant improvements in all PLP metrics. PLP decreased from pre-treatment to last treatment session by $47 \%$ ( $\sigma=39 \%$; absolute $\bar{x}=1 \cdot 0, \sigma=0 \cdot 8 ; \mathrm{p}=0.001)$ for WPD, 32\% ( $\sigma=38 \%$; absolute $\bar{x}=1 \cdot 6, \sigma=1 \cdot 8 ; \mathrm{p}=0.007)$ for NRS, and 51\% ( $\sigma=33$; absolute $\bar{x}=9 \cdot 6, \sigma=8 \cdot 1 ; p=0.0001)$ for PRI. The NRS for intrusion of pain in activities of the daily living and 
sleep was reduced by $43 \%(\sigma=37 \%$; absolute $\bar{x}=2 \cdot 4, \sigma=2 \cdot 3 ; \mathrm{p}=0.004)$ and $61 \%(\sigma=39 \%$; absolute $\bar{x}=2 \cdot 3$, $\sigma=1 \cdot 8 ; \mathrm{p}=0.001)$, respectively. Two out of four patients who were on medication reduced their intake in grams by $81 \%$ and $33 \%$. Improvements remained 6 months after the last treatment.

Interpretation: Our findings suggest potential value in motor execution of the phantom limb as a treatment for PLP. Promotion of phantom motor execution aided by machine learning, AR/VR, and gaming, is a non-invasive, non-pharmacological and engaging treatment with no identified side effects at present.

Funding: VINNOVA, Promobilia foundation, Jimmy Dahlstens Fond, PicoSolve, and Innovationskontor Väst.

\section{Introduction}

In addition to the functional challenges caused by the amputation of an extremity, patients often develop painful sensations perceived as originating from the missing limb, hence the name, phantom limb pain (PLP). Although central and peripheral factors have been implicated in the genesis of such neuropathic pain, the former is believed to be the major contributor. ${ }^{1,2}$ Flor et al. showed that PLP is closely related to neuroplastic changes in at least the primary somatosensory cortex. ${ }^{3}$ Whereas this finding has emerged repeatedly in studies by Flor's group ${ }^{4-6}$ and others, ${ }^{7}$ Makin et al. found that rather than cortical reorganization, reduced inter-hemispheric functional connectivity might be the major contributor to PLP. ${ }^{8}$ In either case, these findings suggest central malplasticity as responsible for maintaining PLP. Neuroplasticity-based approaches for the relief of PLP, such as motor imagery and mirror therapy, ultimately aim to regain brain circuitry from pain. Here, we investigated a novel approach that overcomes methodological limitations of previous treatments by ensuring that central and peripheral mechanisms in motor control are activated during the therapy.

Motor imagery along with meditation has been found to normalize previously altered cortical maps and reduced PLP. ${ }^{7}$ However, motor imagery was also found to increase pain in randomized clinical studies. ${ }^{9,10}$ These findings led to the suggestion that motor imagery should not be used alone but in combination with other treatments, such as mirror therapy. ${ }^{11}$ Mirror therapy by itself has been shown to be more effective than motor imagery, ${ }^{9,10}$ and although it is often argued that similar brain areas are activated in motor imagery and execution, the degree of activation is not the same. ${ }^{12}$ Recently, excitatory coupling between thalamus and primary motor cortex was found necessary for motor execution, but not for motor imagery..$^{13}$ The difference in activation networks between motor execution and imagery extends to the peripheral circuitry. These findings led us to hypothesize that true motor execution of the phantom would provide a more integral normalization (cortical, sub-cortical and peripheral circuits), and therefore potentially relieve pain in patients for whom conventional approaches have failed.

We have shown the feasibility of decoding the execution of phantom movements (motor volition of the missing limb) using patterns of myoelectric activity at the stump, despite the fact that the distal muscles originally responsible for such movements are lost due to the amputation. ${ }^{14,15}$ For example, the synergistic muscular activation at the stump of a transhumeral amputee produce distinctive patterns for 
different phantom hand movements, thus making it possible for machine learning algorithms to infer motor volition using information from above-elbow musculature. In this way, myoelectric pattern recognition (MPR) allows the direct use of phantom movements in rehabilitation tasks purposely based in motor execution of the phantom limb, further referred to as phantom motor execution.

Visual feedback has been found to facilitate phantom movements ${ }^{16}$ and it can potentially induce the illusion of a restored body representation. Therefore, we have combined MPR with Augmented Reality (AR) to provide appropriate and timely visual feedback. Using a conventional webcam and monitor, patients can observe themselves with a virtual arm in the anatomically correct location of the missing arm. Congruent location and orientation of the limb is known to be fundamental for perceptual illusions. ${ }^{17}$ A fiducial marker in the patient's stump provides guidance for moment to moment positioning of the virtual arm. Therefore, the patient can move freely while preserving the virtual arm in the anatomically correct placement. MPR then allows the virtual arm to respond to individual or simultaneous phantom movements under control of the patient. ${ }^{18}$ In this approach, visual feedback not only serves to provide a visual illusion of body completeness, but also informs the motions resulting from patterns of muscular activation, patterns that the patient is dynamically adapting to achieve a variety of movements. In addition, using MPR for control and virtual environments for visualization allows engaging therapeutic tasks ("serious gaming") to be implemented in order to promote phantom motor execution. This concept was first introduced in the case study of one patient with intractable chronic $\mathrm{PLP},{ }^{14}$ the results of which motivated the current multi-center study in similar chronic PLP suffers for whom no other approach had been effective.

The therapy proposed here (phantom motor execution) deviates from the mirror concept due to its independence from the contralateral limb, which also makes it equally valid for bilateral amputees. The main difference relies on requiring appropriate muscular activation in the affected limb, as opposed to using the healthy contralateral. Phantom motor execution and mirror therapy request patients to perform movements with the phantom limb, however, in only the former the actual execution of movement is an inherent component necessary for the treatment to take place. In mirror therapy, it is enough for the patient to move their healthy arm to produce movement in the reflected limb, however whether the patient is actually engaging the appropriate brain areas in execution of phantom movements is unknown.

Previous approaches based on virtual or augmented reality use cameras or instrumented gloves in the able contralateral limb, ${ }^{19-21}$ which makes these solutions methodologically equivalent to mirror therapy. Motion tracking systems have been suggested as an alternative source for control, ${ }^{22}$ and although in this case the affected limb is used to provide the location of the virtual one, important distal movements of the phantom limb (e.g. hand open) cannot be inferred using said technology. Overall, the effectiveness of previous virtual approaches have been moderate and limited to case studies with short-term followup (two or four weeks), ${ }^{20,21}$ with a single larger study of 14 patients with no follow-up. ${ }^{22}$ In the present study, follow-up was performed at one, three, and six months after the last treatment session. 


\section{Methods}

\section{Participants}

Fourteen individuals with upper limb amputation afflicted by refractory chronic PLP participated in this study (Table 1 ). Their mean $(\bar{x})$ age was 50 years old (median $\tilde{x}=52$, standard deviation $\sigma=14$, range $R=26$ 74). They have experienced PLP since soon after their amputation in average ten years at inclusion $(\tilde{x}=5$, $\sigma=11, R=2-36)$. Patients were treated previously with at least one clinically used method with no beneficial outcome for an average of six years after their last treatment and before inclusion ( $\tilde{x}=3, \sigma=7$, $R=0 \cdot 1-22$ ). The level of amputation was equally transhumeral and transradial. Two of the patients were bilateral amputees but only the limb with highest pain was treated in order to keep an equivalent administration regime. Ethical approval for this study was granted by the ethical committees of Västra Götalandsregionen in Sweden, and University Rehabilitation Institute in Slovenia. All subjects provided written informed consent prior to inclusion. Subjects were told about the possibility of a transitory pain increment as observed in previous work. ${ }^{14}$ They were also informed about the research nature of this procedure where the outcome would be uncertain. This was done to reduce potential placebo effects due to expectation. ${ }^{23}$

\begin{tabular}{|c|c|c|c|c|c|c|c|c|c|}
\hline $\begin{array}{l}\text { Amp. } \\
\text { cause }\end{array}$ & $\begin{array}{l}\text { Amp. } \\
\text { level }\end{array}$ & $\begin{array}{l}\text { Age } \\
\text { (yrs.) }\end{array}$ & $\begin{array}{c}\text { Amp. } \\
\text { time (yrs.) }\end{array}$ & $\begin{array}{c}\text { PLP } \\
\text { time (yrs.) }\end{array}$ & $\begin{array}{l}\text { PLP (NRS) } \\
\text { Pre-treat. }\end{array}$ & $\begin{array}{c}\text { Previous } \\
\text { treatments }\end{array}$ & $\begin{array}{l}\text { PTLS } \\
\text { (yrs.) }\end{array}$ & $\begin{array}{l}\text { Current } \\
\text { med. }\end{array}$ & $\begin{array}{c}\text { Med. } \\
\text { time (yrs.) }\end{array}$ \\
\hline Trauma & $\mathrm{TH}$ & 30 & 1.9 & $1 \cdot 8$ & 4 & MT, TENS, Pharma. & 0.6 & - & - \\
\hline Trauma & $\mathrm{TR}$ & 26 & $4 \cdot 4$ & $4 \cdot 3$ & 3 & MT, TENS, Pharma. & $3 \cdot 9$ & - & - \\
\hline Trauma & TR & 49 & $4 \cdot 3$ & $3 \cdot 7$ & 7 & MT, Imp. Nstim., Pharma. & $4 \cdot 1$ & - & - \\
\hline Infection & $\mathrm{TH}-\mathrm{Bi}$ & 74 & $10 \cdot 2$ & $10 \cdot 0$ & 5 & MT, TENS, Acup., Pharma. & $2 \cdot 1$ & Gabapentin & $3 \cdot 8$ \\
\hline Trauma & $\mathrm{TH}$ & 62 & $36 \cdot 3$ & $36 \cdot 3$ & 5 & MT, Acup., Pharma. & $0 \cdot 1$ & - & - \\
\hline Trauma & $\mathrm{TR}$ & 52 & $3 \cdot 3$ & $3 \cdot 2$ & 8 & MT, TENS, Pharma. & $1 \cdot 8$ & Morphine & $2 \cdot 7$ \\
\hline Trauma & $\mathrm{TH}$ & 60 & $31 \cdot 2$ & $31 \cdot 0$ & 5 & MT, Acup., Medit., Med. & $19 \cdot 1$ & - & - \\
\hline Trauma & $\mathrm{TH}-\mathrm{Bi}$ & 56 & $25 \cdot 4$ & $25 \cdot 0$ & 6 & TENS, Acup., Pharma. & $21 \cdot 8$ & - & - \\
\hline Trauma & TR & 51 & 4.9 & $4 \cdot 5$ & 5 & $\mathrm{MT}$ & $5 \cdot 3$ & - & - \\
\hline Trauma & $\mathrm{TR}$ & 69 & $5 \cdot 7$ & $5 \cdot 4$ & 2 & Heat, Pharma. & $5 \cdot 4$ & - & - \\
\hline Trauma & $\mathrm{TH}$ & 28 & $3 \cdot 6$ & $3 \cdot 6$ & 6 & TENS, Snoezelen, Pharma. & $2 \cdot 9$ & Lyrica & $2 \cdot 4$ \\
\hline Tumor & $\mathrm{TH}$ & 48 & $2 \cdot 0$ & $2 \cdot 0$ & 4 & TENS, Pharma. & $0 \cdot 3$ & Lyrica & $2 \cdot 0$ \\
\hline Trauma & TR & 52 & $3 \cdot 8$ & $3 \cdot 8$ & 6 & TENS, Pharma. & $2 \cdot 0$ & - & - \\
\hline Trauma & $\mathrm{TR}$ & 47 & $9 \cdot 3$ & $9 \cdot 3$ & 7 & TENS, Pharma. & $9 \cdot 2$ & - & - \\
\hline \multicolumn{2}{|l|}{ Mean $(\bar{x})$} & $50 \cdot 3$ & $10 \cdot 4$ & $10 \cdot 3$ & $5 \cdot 2$ & & $5 \cdot 6$ & & $2 \cdot 7$ \\
\hline \multicolumn{2}{|c|}{ Standard Dev. $(\sigma)$} & $13 \cdot 9$ & $11 \cdot 1$ & $11 \cdot 1$ & $1 \cdot 6$ & & $6 \cdot 5$ & & 0.7 \\
\hline \multicolumn{2}{|c|}{ Median $(\tilde{x})$} & $51 \cdot 5$ & $4 \cdot 7$ & $4 \cdot 4$ & $5 \cdot 0$ & & $3 \cdot 4$ & & $2 \cdot 5$ \\
\hline \multicolumn{10}{|c|}{$\begin{array}{l}\text { Abbreviations: amputation (Amp.), transhumeral (TH), transradial (TR), bilateral (Bi), phantom limb pain (PLP), numeric rating scale (NRS), treatment } \\
\text { (treat.), mirror therapy (MT), transcutaneous electrical nerve stimulation (TENS), medications (Pharma.), implanted neurostimulator (Imp. Nstim.), } \\
\text { acupuncture (Acup.), meditation (Medit.), previous treatment last session (PTLS). Snoezelen refers to multisensory stimulation therapy, which does not }\end{array}$} \\
\hline
\end{tabular}

\section{Evaluation metrics}

The pain evaluation variables included the following: the numeric rating scale (NRS, 0 "none" to 10 "maximum"), used as a common tool to evaluate the intensity of pain at present; the pain rating index (PRI, 0 to 75) formed by the summed contribution of 15 qualities of pain as in the short-form McGill Pain Questionnaire, ${ }^{24}$ and scored individually using the present pain intensity scale (PPI, ${ }^{25} 0$ "none" to 5 "excruciating"); the weighted pain distribution (WPD, ${ }^{14} 0$ "none" to 5 "maximum") aimed to capture the time-varying nature of chronic pain by adding the contributions of weighted portions of time spent in six pain levels $\left(\mathrm{PP}^{25}\right)$; and pain frequency was measured using a study-specific descriptive scale of seven steps: 'never', 'once per month', 'once per week', 'few times per week', 'once per day', 'few times per day', and 'always'. In addition, the intrusion of PLP in activities of daily living (ADL) and sleep was 
measured by one question each, and scored on the NRS. Changes in prosthetic hardware and medication were monitored during the study. Statistical significance in quantitative measures were calculated using the Wilcoxon Signed-Rank test, and changes in the incidence of the different qualities of pain were evaluated by the Sign test (see extended methods).

An important methodological feature in our evaluation of pain was to conduct the interview before each treatment session. This had the purpose of capturing the analgesic effects at a longer term (between sessions), as opposed to immediately after the intervention when the highest improvement is known to peak thus biasing the interpretation of clinical relevance. We considered this to be of particular importance when treating chronic conditions such as PLP.

\section{Interventions}

Patients with known chronic intractable pain were recruited at three clinics in Sweden and one in Slovenia: Sahlgrenska University Hospital in Gothenburg, Örebro University Hospital in Örebro, BräckeDiakoni Rehabcenter Sfären in Stockholm, and the University Rehabilitation Institute in Ljubljana. Therapists at these clinics were introduced to the technology with one practical demonstration and were monitored by at least the lead author during the first intervention of their first patient. The therapists conducted the rest of the interventions independently following the study protocol and returned a signed case report form at the end of the study.

All patients received an intervention twice per week except for one who had it daily. Each session lasted two hours and consisted of 1) pain evaluation, 2) placement of the electrodes and fiducial marker, 3) practice motor execution in AR, 4) gaming by racing car using phantom movements, and 5) matching random target postures of a virtual arm in VR (Figure 1 and Video 1). Steps 3 to 5 were repeated for different movements following three levels of difficulty: A) two movements forming one degree of freedom (DoF); B) two to four DoF; and C) two or more DoF simultaneously. Clinicians were instructed to advance the level of difficulty once the previous level was accomplished successfully, and revert to the previous level if the patient showed considerable difficulty accomplishing the tasks (see extended methods). This was done to keep the phantom motor execution challenging but feasible as it is known that mental effort is required for plasticity to take place. The treatment consisted of 12 sessions and follow-up interviews at one, three, and six months after the last session.

A user-friendly system was developed for independent use at the clinics (software and hardware). This system named Neuromotus was based on the open source platform BioPatRec, ${ }^{26}$ where algorithms for the prediction of individual and simultaneous movements ${ }^{18}$ are implemented together with virtual environments and gaming control interfaces. 


\section{Role of the funding source}

The funders had no role in study design, data collection, data analysis, data interpretation, or writing of the report. The corresponding authors had full access to all the data in this study and had final responsibility for the decision to submit for publication.

\section{Results}

Overall continuous reduction of PLP was measured by all metrics (Figure 2). Average improvement at the last treatment session was recorded by the WPD (relative $\bar{x}=47 \%, \sigma=39 \%$; absolute $\bar{x}=1 \cdot 0, \sigma=0.8$; $\mathrm{p}=0 \cdot 001$ ), NRS (relative $\bar{x}=32 \%, \sigma=38 \%$; absolute $\bar{x}=1 \cdot 6, \sigma=1 \cdot 8 ; \mathrm{p}=0 \cdot 007$ ), and PRI (relative $\bar{x}=51 \%, \sigma=33 \%$; absolute $\bar{x}=9 \cdot 6, \sigma=8 \cdot 1 ; \mathrm{p}=0 \cdot 0001$ ). Reduction in intensity and quality of pain was found in all patients (PRI; $\bar{x}=51 \%, \sigma=33 \% ; \mathrm{p}=0.0001$ ); a positive change in the time-intensity profile was found in 12 patients (WPD; $56 \%, \sigma=35 \% ; \mathrm{p}=0.001$ ); and, reduction of pain intensity at present was found in nine patients (NRS; $55 \%$, $\sigma=27 \% ; \mathrm{p}=0.004)$. Additionally, reduction in NRS of at least two points was found in eight patients.

Improvements in time-variation (WPD) and pain intensity at present (NRS) were maintained at all of the follow-ups. The average improvement measured by PRI at the last treatment session decreased by $2 \%$, $6 \%$, and $24 \%$ at one, three, and six month follow-ups, respectively (Figure 2 ).

Reduction in PLP was also found by the change in the number of patients reporting fifteen different qualities of pain (PRI) before and after treatment (Figure 3). Thirteen of such pain qualities showed reduced occurrence in the population. "Stabbing" and "tiring-exhausting" were found significantly less prevalent after treatment $(p=0 \cdot 016)$.

Thirteen patients reported that PLP interfered with their ADL and sleep at inclusion (NRS); such intrusion was reduced on average by $43 \%$ ( $\sigma=37 \%$; absolute $\bar{x}=2 \cdot 4, \sigma=2.3 ; \mathrm{p}=0.004)$ and $61 \%(\sigma=39 \%$; absolute $\bar{x}=2 \cdot 3, \sigma=1.8 ; p=0.001$ ), respectively (Figure 4). After treatment, eight patients (62\%) reported less interference in ADL by at least two points in NRS (representing a reduction of $\bar{x}=67 \%, \sigma=26 \%, p=0.004$ ). Similarly, 11 patients (85\%) reported less interference in sleep by an average of $72 \%(\sigma=32 \%, p=0.001)$. The improvements remained at the one, three, and six month follow-ups (Figure 4). In addition, the intake of pain medication was reduced by $81 \%$ and $33 \%$ grams in two out of four patients who were continuously medicated for at least two years (reduction relative to the initial dose).

The profile of PLP frequency showed a positive change after treatment and at all follow-ups (Figure 5). One patient was unavailable for the third month follow-up, and a different one for the sixth month follow-up (computations were done accordingly).

Statistically significant correlations of moderate strength were found between WPD (duration and intensity), PRI (quality and intensity), and NRS (present intensity), as well as in the intrusion of PLP in the patients' ADL and sleep ( $p<0 \cdot 0001$ ), Figure 6 (online). Normalized changes in WPD, NRS, PRI, intrusions in $\mathrm{ADL}$ and sleep along the treatment and follow-ups are shown in Figure 6 (online). 


\section{Discussion}

In this study we examined the effectiveness of a novel therapy for PLP based on the promotion of phantom motor execution. This non-invasive approach exploits principles of brain plasticity reduced PLP by approximately $50 \%$ in chronic sufferers from whom conventional treatments failed. Moreover, the intrusion of PLP in ADL and sleep was also reduced by an average of $50 \%$.

All the patients included in this study were first treated with other methods for a significant amount of time prior to phantom motor execution ( $\bar{x}=6$ years). It is thus reasonable to expect little to no carry over effects from previous therapies. Similarly, since this group of patients had suffered from chronic PLP for an average of ten years, pain relief owing to "natural history" or "regression to the mean" effects is unlikely. In addition, patients who were under medication had already been on it for over two years, they had no increase of dosage during the study, and therefore pain changes due to medications are also unlikely. Placebo effects cannot be disentangled in this study, however, the persistent relief of PLP after six months makes it less likely to be caused by such effects. Nevertheless, none of the aforementioned alternative explanations can be fully rejected by the evidence presented in this study due the lack of a control group. An additional limitation of this study is that the follow-ups were conducted by the same clinicians who administered the interventions, which can be regarded as potential source of bias.

A drawback of the technology proposed here is that volitional control of musculature at the stump is necessary, thus patients with nerve injuries where no muscular activity can be elicited cannot utilize this technology. Similarly, patients with shoulder disarticulation may not have sufficient musculature to allow for the prediction of distal movements unless recipients of targeted muscle reinnervation. ${ }^{27}$ Recording of weak muscular contractions is technologically feasible; however, the extent at which such activity can be used in this treatment must be determined for each individual case, particularly when excessive soft, fat, and scar tissues are present. In this study, patients with high transhumeral amputations were treated with the inclusion criteria that at least a portion of the biceps or triceps muscles were viable. Appropriate motor volition of the phantom hand in a shoulder disarticulation might not be possible with the present approach, but this must be investigated further.

\section{Pain improvement}

Reduction of pain by $50 \%$ or two points in NRS have been suggested as clinically relevant outcomes. ${ }^{28}$ The improvements found in this study on intractable chronic PLP suffers were of approximately 50\%, and more than half of the patients improved by at least two points in NRS. Different pain measures were used to capture the complex profile of chronic PLP considering intensity, duration, frequency, quality and intrusion. Continuous improvement was measured in all these metrics for the twelve interventions, and was still observable at the last follow-up session. It is therefore arguable that a longer treatment regime (more sessions) would further decrease PLP, particularly considering that these patients had spent an average of ten years in a maladapted painful state.

All but one patient reported a perceived improvement in their PLP state relative to prior treatment. This patient reported a stressful life situation during the study period, which he believed interfered with our treatment attempts. Situational stress is known to be strongly related with PLP ${ }^{29}$, and therefore was potentially the cause of the poor improvement. Arguably, the treatment might have prevented the 
exacerbation of PLP during this period of stress. The patient requested to be treated again once his personal problems are resolved. The patient who secondly least benefited from the treatment reported no change in his sustained pain (NRS) but showed improvement in PRI and the intrusion of PLP in ADL and sleep. The major benefit for this patient was the disappearance of "flare-ups" (short periods of high pain intensity), which allowed him to sleep better. Since the time spent in these high-intensity periods was minimal in comparison with the rest, little difference was captured by the WPD and none by the NRS. The improvement was mostly recorded by the PRI because the "flare-ups" were of specific qualities, thus their contribution to PRI disappeared along with their occurrence. This case highlights the importance of using different pain measures. All patients reported difficulties moving their phantom limb at the beginning of the trial, but were able to accomplish it after few sessions aside from this patient, who continue reporting considerable difficulties to open and close his phantom hand.

\section{Relation to non-pharmaceutical therapies}

Non-pharmacological approaches exploiting brain plasticity such as mirror therapy or motor imagery have shown promising results. However, despite their simplicity, low-cost, and clinical evidence, these therapies have not ultimately solved PLP. A common drawback of these approaches is the unchallenging repetitive nature of the exercises where no timely feedback is provided, mostly because the movement of the phantom limb cannot be monitored.

Promotion of motor execution is a fundamental part of Constraint-Induced Movement Therapy (CIMT), a method which has been successful in neuromuscular rehabilitation after stroke, multiple sclerosis, and traumatic brain injuries. ${ }^{30}$ In CIMT, the able limb is restrained to force the patient to utilize the affected one. This strategy has been proven effective but is criticized for the strain placed on the patients. In the approach proposed here (MPR + AR/VR + gaming), the affected limb is forced to be activated but in contrast to the CIMT, is pleasant and entertaining.

Graded motor imagery (GMI) which combines lateralization, motor imagery and mirror therapy has shown promising results in PLP and CRPS. ${ }^{31}$ The approach proposed here can potentially improve GMI further by making sure that motor execution takes place. GMI seems reasonable for patients where kinesiophobia is present due to its graded component, as well as for patients where motor imagery alone is perceived as painful. In the current study, patients did not report fear of movement nor pain related to imagination, intention, or any motor related actions. Negative results in the clinical implementation of GMI had been attributed to less therapist-patient contact and practice, ${ }^{32}$ which stresses the importance of proper translation and dissemination of therapies. In our case, we found that the design of our device allowed an easy translation to new centers requiring a single visit for instruction. In addition, if patients are asked to practice independently at home or clinic, the clinician can easily verify the time the patients spent using the system, thus improvement can be confidently coupled to therapy exposure and frequency. Moreover, gamification via AR/VR makes the approach proposed here more engaging than mirror therapy, GMI and CIMT.

The technology presented in this study allows for an integrated treatment-evaluation system. In addition, it is potentially applicable to other conditions such as hemiparesis after stroke, impaired motor 
control due to nerve injuries, or recovery after hand surgery. In these cases, functional restoration could be improved by increasing neural drive to muscles.

\section{Potential working mechanisms}

Owing to the functional link found between cortical reorganization and PLP, therapeutic approaches aiming to alter the former will likely impact the latter. ${ }^{33}$ Explanations for cortical reorganization after amputation often emphasize sensory over motor deprivation as the principal cause. However, the motor cortex is equally affected (disused) and as opposed to incomplete sensory stimulation, a major part of appropriate motor related areas can still be engaged to produce movements in the missing limb. Appropriate stimulation of the sensory cortex would require a phantom map of referred sensations (rarely present and often incomplete), targeted sensory reinnervation, or implantation of neural interfaces for the direct stimulation of afferent fibers, which can only deliver limited qualities and locations at present. ${ }^{15,34}$ Conversely, motor execution of complex movements can be promoted noninvasively with the approach proposed here, which also includes sensory (visual) and psychological (attentional) components.

Patients were notably paying considerable attention to their stump during the first sessions, mostly to the afferent excitation resulting from muscular contraction while trying to modulate motor output to achieve a correct virtual movement. This might have also contributed to PLP relief via competitive plasticity as suggested in sensory discrimination approaches. ${ }^{35}$ In addition, ownership and agency of the virtual models might had contributed to pain relief, however, these were not explicitly measured and the effect was potentially variable in the different scenarios (AR versus gaming). The effect of nonpharmacological perceptual approaches to PLP have mostly been attributed to appropriate sensory feedback, which is limited to visual input since natural tactile and proprioceptive information is not provided. However, despite that visual feedback has shown to facilitate phantom movements, it has also been found not absolutely required for PLP relief. ${ }^{16}$ Carefully designed studies are still necessary to determine the contribution of each of these elements to pain relief.

The hypothesis of motor-sensory incongruence states that the mismatch between motor intention, proprioception, and visual feedback, might be the cause of PLP in a similar way that visual-vestibular incongruences cause motion sickness. Based on this theory, Harris suggested that emphasizing the use of appropriate visual feedback would increase the effectives of pathological pain therapies. ${ }^{36}$ Our findings do not directly support this hypothesis since augmented reality, allegedly the most immersive situation, was only one third of the therapy. The common denominator at all stages was considerable phantom motor execution monitored in real-time with visual feedback in various forms. i.e., controlling the racing game did not require physiologically appropriate commands, but yet demanded distinctively different phantom movements.

Similarly to CIMT, the basic mechanism of phantom motor execution forces the patient to recruit the necessary brain circuitry for the production of movement. CIMT cannot be used in PLP for obvious reasons. However, our technology does a similar job of restraining the healthy limb and forcing the use of the affected one with the advantage of being pleasant and entertaining. Therefore, this technology 
(MPR + AR-VR + gaming) can potentially be used to further improve CIMT or as a rehabilitation tool on its own.

Cortical re-organization due to amputation is commonly explained by the effect of deafferentation, but it must not be forgotten that motor execution is equally affected. This treatment exploits the contribution of central and peripheral motor circuitry to reverse maladapted changes. The underlying mechanisms for which our phantom motor execution, mirror therapy, motor imagery, and GMI reduce PLP are poorly understood, but these approaches are all based in principles of brain plasticity. Further work is necessary to identify functional and structural changes caused by pain and by the intervention itself. From the clinical view point, additional clinical evidence is necessary to prove the efficacy of the approach proposed here.

\section{Conclusion}

Phantom limb pain (PLP) is a difficult condition to treat that can seriously hinder patients' quality of life. Surgical and pharmacological approaches have fallen short of providing an effective treatment while yielding considerable side effects. The results from this study suggest potential value in motor execution of the phantom limb, as a non-invasive and non-pharmacological treatment for PLP. Promotion of phantom motor execution aided by myoelectric pattern recognition, AR/VR, and gaming, is an engaging treatment which was found to reduce chronic PLP, with no identified side effects, in a group of patients for whom conventional treatments failed. Additional randomized clinical trials are necessary to rigorously prove the efficacy of this approach.

\section{Contributors}

MOC conceived the treatment, developed the algorithms for the prediction of motor volition, analyzed the data, reviewed the literature, and draft the manuscript. MOC and RAG designed the study. RAG coordinated the study. MBK developed the AR technology and software. AZE and MOC developed the hardware. $\mathrm{KCW}, \mathrm{KKO}, \mathrm{CW}, \mathrm{KE}, \mathrm{AS}, \mathrm{CR}, \mathrm{ZP}, \mathrm{HB}$, and $\mathrm{LH}$ conducted the interventions and collected the data. RAG and KKO consolidated the data. HB and LH revised the manuscript. All authors revised the study protocol and collected data, and approved the final report.

\section{Declaration of interest}

MOC, RAG, MBK, and AZE were partially funded by Integrum $A B$, a for-profit organization which might commercialize an improved version of the technology here described. The core technology used in this study (machine learning and virtual reality) has been made freely available as open source by MOC. KCW, $\mathrm{KKO}, \mathrm{CW}, \mathrm{KE}, \mathrm{AS}, \mathrm{CR}, \mathrm{ZP}, \mathrm{HB}$, and LH declare no competing interests.

\section{Acknowledgments}

This study was funded by VINNOVA, Jimmy Dahlstens Fond, PicoSolve, and Innovationskontor Väst. We acknowledge the patients who participated in this study for their time and effort. AS and CR thank 
Bräcke Diakoni for allowing them to participate in this study. We thank Bo Håkansson and Rickard Brånemark for their support during this research.

\section{Bibliography}

$1 \quad$ Nikolajsen L, Jensen TS. Phantom limb pain. Br J Anaesth 2001; 87: 107-16.

2 Flor H. Phantom-limb pain: characteristics, causes, and treatment. Lancet Neurol 2002; 1: 182-9.

3 Flor $\mathrm{H}$, Elbert T, Knecht $\mathrm{S}$, et al. Phantom-limb pain as a perceptual correlate of cortical reorganization following arm amputation. Nature 1995; 375: 482-4.

4 Knecht S, Henningsen $\mathrm{H}$, Höhling $\mathrm{C}$, et al. Plasticity of plasticity? Changes in the pattern of perceptual correlates of reorganization after amputation. Brain 1998; 121: 717-24.

5 Lotze M, Grodd W, Birbaumer N, Erb M, Huse E, Flor H. Does use of a myoelectric prosthesis prevent cortical reorganization and phantom limb pain? Nat Neurosci 1999; 2: 501-2.

6 Grüsser SM, Winter C, Mühlnickel W, et al. The relationship of perceptual phenomena and cortical reorganization in upper extremity amputees. Neuroscience 2001; 102: 263-72.

7 Maclver K, Lloyd DM, Kelly S, Roberts N, Nurmikko T. Phantom limb pain, cortical reorganization and the therapeutic effect of mental imagery. Brain 2008; 131: 2181-91.

8 Makin TR, Scholz J, Filippini N, Henderson Slater D, Tracey I, Johansen-Berg H. Phantom pain is associated with preserved structure and function in the former hand area. Nat Commun 2013; 4: 1570.

9 Chan BL, Witt R, Charrow AP, et al. Mirror Therapy for Phantom Limb Pain. N Engl J Med 2007; 357: 2206-7.

10 Cacchio A, De Blasis E, Necozione S, Orio F di, Santilli V. Mirror Therapy for Chronic Complex Regional Pain Syndrome Type 1 and Stroke. N Engl J Med 2009; 361: 634-6.

11 Bowering KJ, Connell NEO, Tabor A, et al. The Effects of Graded Motor Imagery and Its Components on Chronic Pain: A Systematic Review and Meta-Analysis. J Pain 2013; 14: 3-13.

12 Hanakawa T, Immisch I, Toma K, Dimyan MA, Van Gelderen P, Hallett M. Functional properties of brain areas associated with motor execution and imagery. J Neurophysiol 2003; 89: 989-1002.

13 Fernández-Espejo D, Rossit S, Owen AM. A Thalamocortical Mechanism for the Absence of Overt Motor Behavior in Covertly Aware Patients. JAMA Neurol 2015; : 1.

14 Ortiz-Catalan M, Sander N, Kristoffersen MB, Håkansson B, Brånemark R. Treatment of phantom limb pain (PLP) based on augmented reality and gaming controlled by myoelectric pattern recognition : a case study of a chronic PLP patient. Front Neurosci 2014; 8.

DOI:10.3389/fnins.2014.00024.

15 Ortiz-Catalan M, Håkansson B, Brånemark R. An osseointegrated human-machine gateway for long-term sensory feedback and motor control of artificial limbs. Sci Transl Med 2014; 6: 257re6.

16 Brodie EE, Whyte A, Niven $\mathrm{C}$ a. Analgesia through the looking-glass? A randomized controlled trial investigating the effect of viewing a 'virtual' limb upon phantom limb pain, sensation and movement. Eur J Pain 2007; 11: 428-36. 
Ehrsson HH. The Concept of Body Ownership and Its Relation to Multisensory Integration. In: Stein BE, ed. The New Handbook of Multisensory Processes. Cambridge: MIT Press, 2012: 775-92. Ortiz-Catalan M, Håkansson B, Brånemark R. Real-Time and Simultaneous Control of Artificial Limbs Based on Pattern Recognition Algorithms. IEEE Trans Neural Syst Rehabil Eng 2014; 22: 756-64.

Desmond D, O’Neill K, De Paor A, McDarby G, MacLachlan M. Augmenting the reality of phantom limbs: three case studies using an augmented mirror box procedure. J Prosthet Orthot 2006; 18: 74.

Murray C, Patchick E, Pettifer S. Investigating the efficacy of a virtual mirror box in treating phantom limb pain in a sample of chronic sufferers. Int J Disabil Hum Dev 2006; 5: 227-34. Mercier C, Sirigu A. Training with virtual visual feedback to alleviate phantom limb pain. Neurorehabil Neural Repair 2009; 23: 587-94.

22 Cole J, Crowle S, Austwick G, Slater DH. Exploratory findings with virtual reality for phantom limb pain; from stump motion to agency and analgesia. Disabil Rehabil 2009; 31: 846-54. Pollo A, Amanzio M, Arslanian A, Casadio C, Maggi G, Benedetti F. Response expectancies in placebo analgesia and their clinical relevance. Pain 2001; 93: 77-84.

Melzack R. The Short-form McGill Pain Questionnaire. Pain 1987; 30: 191-7.

Melzack R. The McGill Pain Questionnaire: Major properties and scoring methods. Pain 1975; 1: 277-99.

Ortiz-Catalan M, Brånemark R, Håkansson B. BioPatRec: A modular research platform for the control of artificial limbs based on pattern recognition algorithms. Source Code Biol Med 2013; 8. DOI:10.1186/1751-0473-8-11.

27 Kuiken T, Li G, Lock BA, et al. Targeted muscle reinnervation for real-time myoelectric control of multifunction artificial arms. JAMA J Am Med Assoc 2009; 301: 619-28.

Farrar JT, Young JP, LaMoreaux L, Werth JL, Poole RM. Clinical importance of changes in chronic pain intensity measured on an 11-point numerical pain rating scale. Pain 2001; 94: 149-58.

Arena JG, Sherman R a., Bruno GM, Smith JD. The relationship between situational stress and phantom limb pain: Cross-lagged correlational data from six month pain logs. J Psychosom Res 1990; 34: 71-7.

Taub E, Uswatte G, Mark VW. The functional significance of cortical reorganization and the parallel development of $\mathrm{Cl}$ therapy. Front Hum Neurosci 2014; 8: 396.

31 Moseley GL. Graded motor imagery for pathologic pain: A randomized controlled trial. Neurology 2006; 67: 2129-34.

32 Johnson S, Hall J, Barnett $\mathrm{S}$, et al. Using graded motor imagery for complex regional pain syndrome in clinical practice: failure to improve pain. Eur J Pain 2012; 16: 550-61.

Birbaumer N, Lutzenberger W, Montoya P, et al. Effects of regional anesthesia on phantom limb pain are mirrored in changes in cortical reorganization. $J$ Neurosci 1997; 17: 5503-8.

Tan DW, Schiefer MA, Keith MW, Anderson JR, Tyler J, Tyler DJ. A neural interface provides longterm stable natural touch perception. Sci Transl Med 2014; 6: 257ra138. 


\section{Figures}

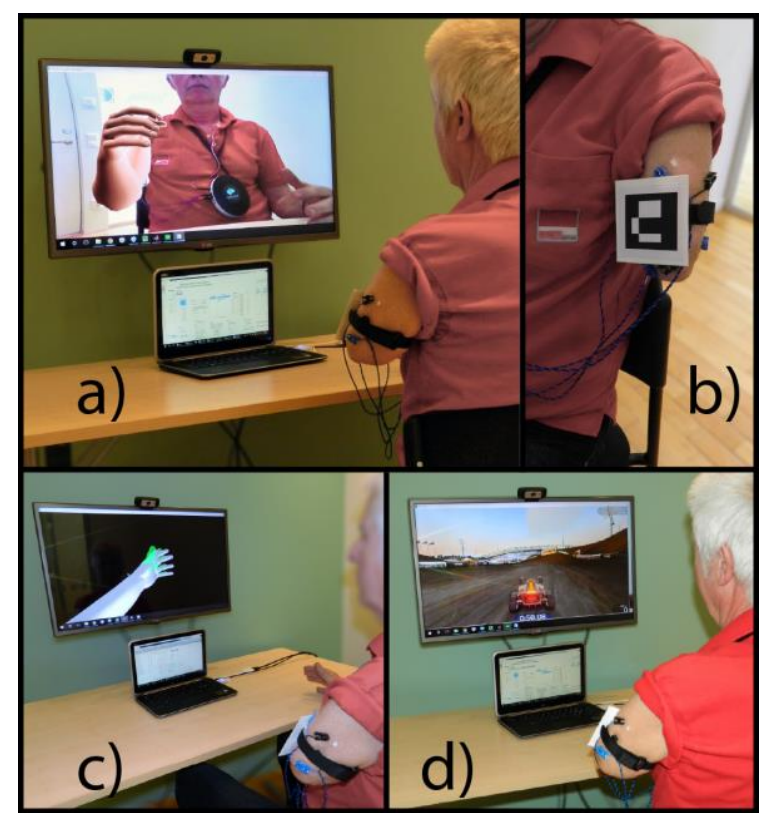

Figure 1. Motor Phantom Execution using MPR, AR/VR, and gaming. a) A conventional webcam provides live video of the patient displayed on a computer screen. A virtual limb is added to the video feed in the location indicated by a fiducial marker (b). Surface electrodes over the stump record synergistic muscles activation during motor volition of the phantom limb (phantom motor execution). Myoelectric pattern recognition is used to decode motor volition and voluntarily control the virtual limb. c) The patient is requested to match random target postures as a rehabilitation task. d) Patient playing a racing game in which the car is driven by phantom movements. See Video 1 for demonstration of a treatment session.
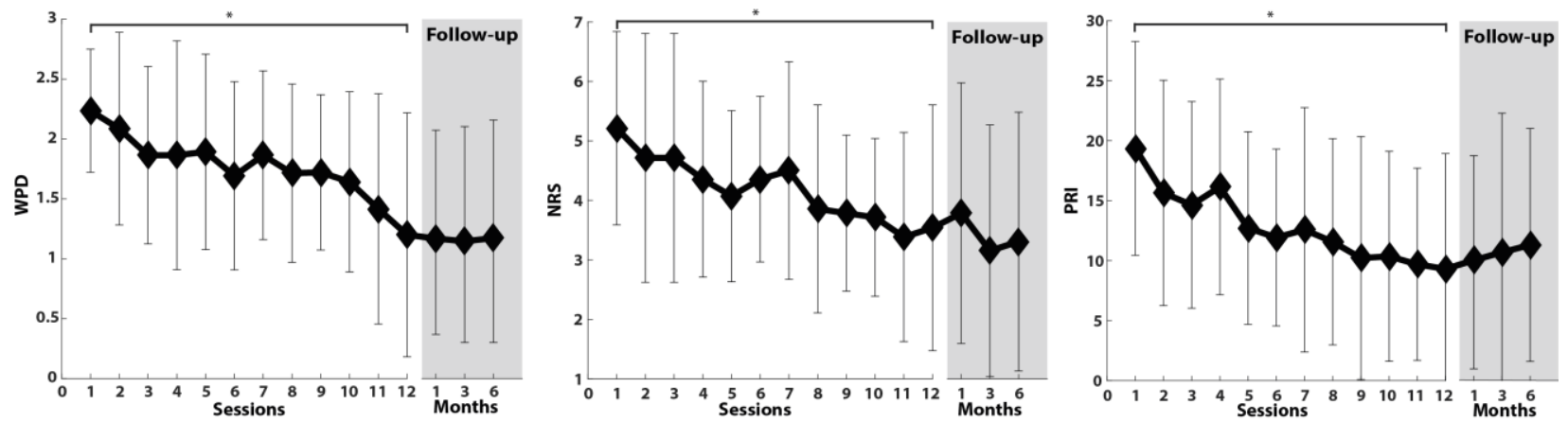

Figure 2. PLP perception during treatment and follow-ups. Insets illustrate the mean of absolute values (markers) with plus/minus one standard deviation (error bars) over 12 sessions and after one, three, and six month follow-ups. Pain metrics used were the weighted pain distribution (WPD), numeric rating scale (NRS), and pain rating index (PRI). Statistical significance between pre-treatment and last treatment session is shown by an asterisk $\left(^{*}\right)$. 
Significance was found at $\mathrm{p}=0.001$ for WPD, $\mathrm{p}=0.007$ for NRS, and $\mathrm{p}=0.0001$ for PRI. The effect size was $1 \cdot 3,0 \cdot 9$, and 1.1 for WPD, NRS, and PRI, respectively. The mean differences (and uncertainty) were $1.0(0 \cdot 8), 1 \cdot 6(1 \cdot 8)$, and 9.6 (8.1) for WPD, NRS, and PRI, respectively.

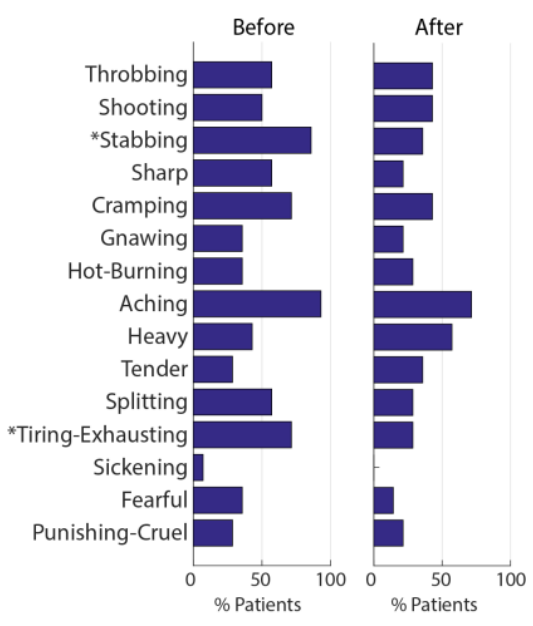

Figure 3. Incidence of pains. The incidence of fifteen qualities of pain as recorded by the Pain Rating Index are presented before and after treatment. Thirteen (87\%) of such pain qualities showed a reduced occurrence in the population after treatment, two of which had a statistically significant change $(\mathrm{p}=0 \cdot 016)$ shown by an asterisk $(*)$.

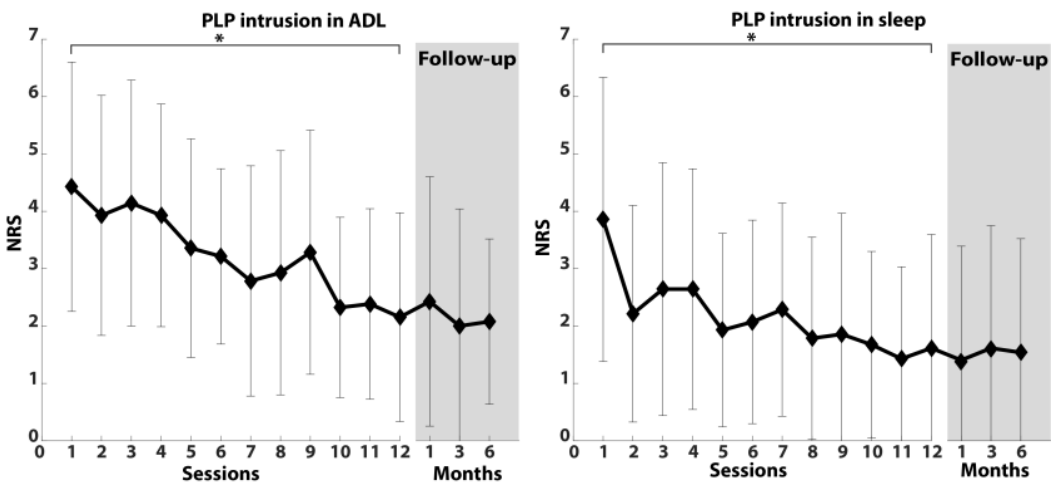

Figure 4. PLP intrusion in ADL and sleep. Markers represent the average PLP intrusion in activities of the daily living (ADL) and during sleep as measured by Numeric Rating Scale (NRS). Error bars show the standard deviation and statistical significance ( $\mathrm{p}=0.004$ for $\mathrm{ADL}$ and $\mathrm{p}=0.001$ for sleep) is shown by an asterisk $\left({ }^{*}\right)$. The effect size was 1.2 for the PLP intrusion in ADL, and 1.0 for intrusion in sleep. The mean differences (and uncertainty) was 2.4 $(2 \cdot 3)$ and $2 \cdot 3(1 \cdot 8)$ for PLP intrusion in ADL and sleep, respectively.

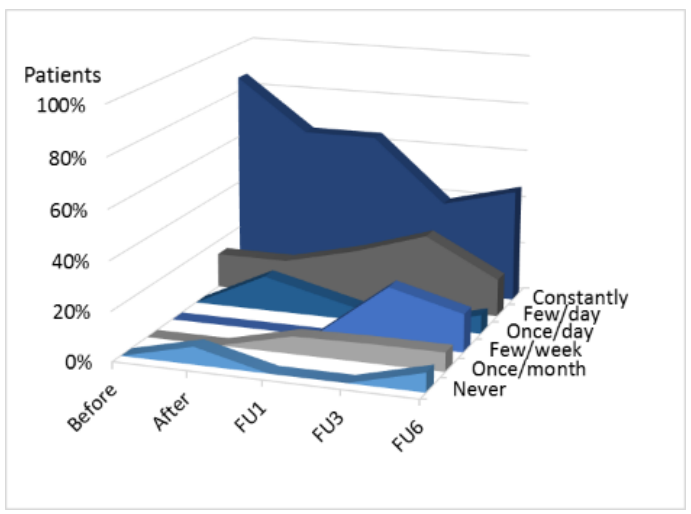


Figure 5. Frequency of PLP perception. Change in the profile of PLP frequency before and after twelve sessions, as well as follow-ups at one, three, and six months (FU1-6). The 'once/week' frequency was removed for clarity as this was not reported.

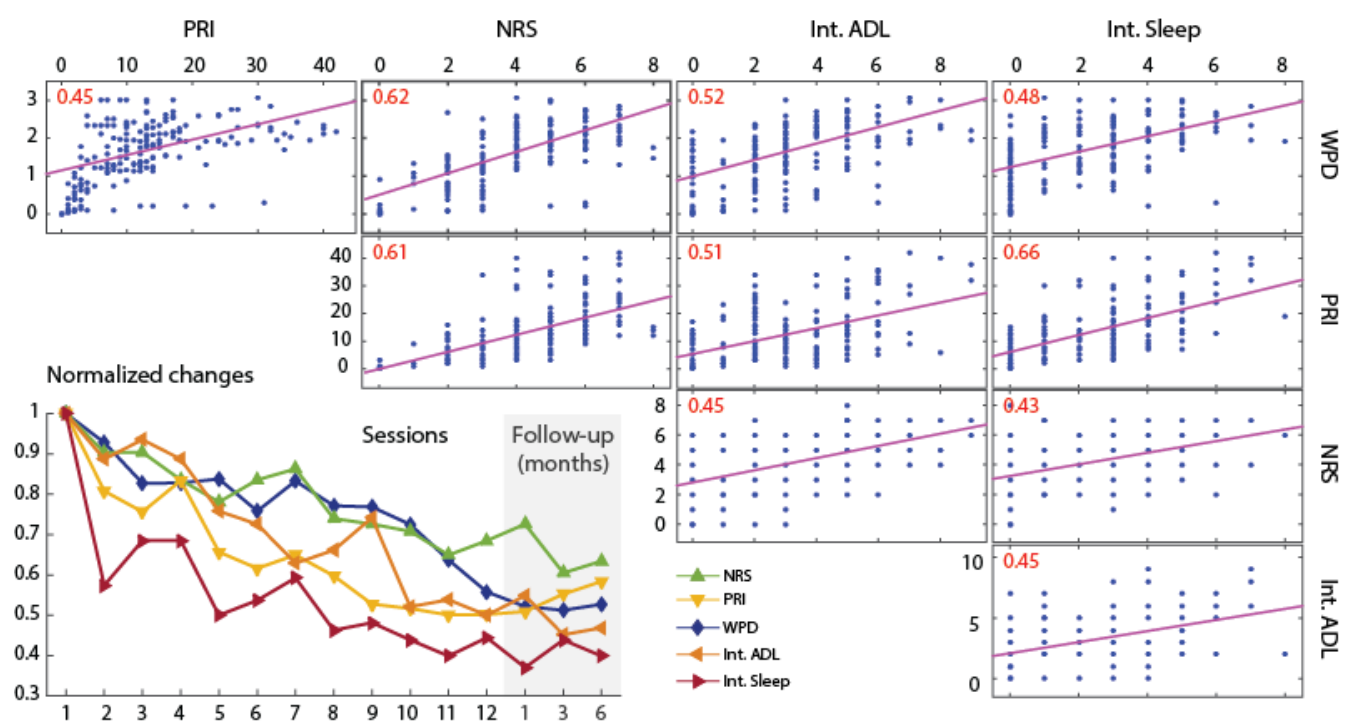

(ONLINE ONLY) Figure 6. Relationships between the different pain measures. Absolute values per subject and session were used to find linear associations among pairs of metrics (rows and columns). The Pearson's correlation coefficients between each metrics are shown in red within each inset (all statistically significant at $\mathrm{p}<0 \cdot 0001$ ), which are also the slopes of the fitting lines. P-values for Pearson's correlation were computed by transforming the correlation to create a $t$ statistic with numObs-2 degrees of freedom. Bottom left inset shows the changes in pain perception as the normalized average to their initial value. The average of absolute values for all subjects was obtained per session, and then normalized to the first session (pre-treatment). 